Poverty Traps and Business Cycles in a Stochastic Overlapping Generations Economy with S-shaped Law of Motion

Schenk-Hoppé, Klaus Reiner

Publication date:

2002

Document version

Early version, also known as pre-print

Citation for published version (APA):

Schenk-Hoppé, K. R. (2002). Poverty Traps and Business Cycles in a Stochastic Overlapping Generations Economy with S-shaped Law of Motion. Department of Economics, University of Copenhagen. 


\section{DISCUSSION PAPERS Department of Economics University of Copenhagen}

\section{$02-13$}

Poverty Traps and Business Cycles in a Stochastic Overlapping Generations Economy with S-shaped Law of Motion

Klaus Reiner Schenk-Hoppé

Studiestræde 6, DK-1455 Copenhagen K., Denmark

Tel. +45 35323082 - Fax +45 35323000

http://www.econ.ku.dk 


\title{
Poverty Traps and Business Cycles in a Stochastic Overlapping Generations Economy with S-shaped Law of Motion
}

\author{
Klaus Reiner Schenk-Hoppé \\ Institute of Economics \\ University of Copenhagen, Denmark \\ klaus@econ.ku.dk
}

October 25, 2002*

\begin{abstract}
This paper contributes to the understanding of stochastic economic dynamics with S-shaped law of motion. Applying random dynamical systems theory, we obtain a complete analysis of a stochastic OLG growth model. In the long-run the economy converges either to a state with no capital (poverty trap) or a sample path of a random fixed point (business cycle). The threshold capital stock separating both regimes is a random variable that depends on the future realization of the shocks; this critical level cannot be identified using past observations. Supply of outside capital therefore has an uncertain effect. Policy recommendations are given which cannot be obtained employing Markov equilibria. A numerical illustration is provided.
\end{abstract}

Keywords: S-shaped stochastic law of motion, random dynamical systems, poverty traps, business cycles, production shocks

JEL-Classification: E32, C62, D91

\section{Introduction}

This paper presents a new approach to the study of stochastic economies with an S-shaped law of motion. Applying random dynamical systems the-

*The first draft of this paper has been written during my stay as visiting scholar at Stanford University. I am grateful to Jens-Ulrich Peter for collaboration on the subject in an early stage. Financial support by the Ecoscientia Stiftung is gratefully acknowledged. 
ory (Arnold 1998), we obtain a comprehensive description of the stochastic short- and long-run dynamics. The central tool in this analysis is the concept of a random fixed point which provides a stochastic analogue of a deterministic fixed point. This notion of a stochastic equilibrium is an alternative to the commonly used Markov equilibrium. The insights obtained by the application of the random fixed point concept cannot be achieved using the Markov equilibrium approach. This is due to the fact that a Markovian equilibrium captures the statistical but not the dynamical properties of the stochastic law of motion. Moreover, existence of Markov equilibria and an exact description of their corresponding stable sets follows readily from our results in the model considered here.

The main goal in this paper is twofold. On the one hand, it explains and illustrates the conceptual and methodological issues of our approach to the study of S-shaped stochastic economic laws. On the other hand, it provides an example of the usefulness of the approach for policy recommendations. We do not strive for generality here. Indeed we will assume specific utility and production functions to make the analysis as transparent as possible. In fact, the dynamics turns out to be considerably more complicated than in economies with concave stochastic law such as the Solow growth model (Schenk-Hoppé and Schmalfuss 2001). To this end, we consider a particular model of an overlapping generations economy with production shocks. The law of motion is an S-shaped function of the capital stock for any realization of the shock. It is proved that only two scenarios can occur in the long-run. The economy converges either to a state with no capital (poverty) or a sample path of a random fixed point (business cycle).

The sets of initial capital stocks leading to one of the regimes are characterized by being lower (poverty) resp. higher (business cycle) than a threshold. The critical level of capital separating both regimes is also a random fixed point (whose existence is also proved here). However, this random fixed point is unstable and depends on the future realization of the shocks. Its state in any period in time therefore cannot be determined using past observations. As a consequence we obtain the result that the supply of outside capital has an uncertain effect. Indeed, the probability of an intervention through a lump-sum transfer of outside capital being successful is determined by the distribution of the unstable random fixed point. It is important to emphasize that this distribution is not a Markov equilibrium (except in the deterministic case).

Our analysis demonstrates that a precise prediction of the effect of capital supply is possible, whereas the Markov equilibrium approach provides only limited information. A numerical illustration of this feature is provided. 
The study of related stochastic overlapping generations models from the Markov equilibrium view is due to Wang $(1993,1994)$. Deterministic models with S-shaped law are studied for instance by Azariadis and Drazen (1990). In their paper existence of a poverty trap (referred to as development trap) and sustainability of higher levels of capital is due to an increasing returnsto-scale technology and insufficient productivity for small capital stocks. We assume a constant returns-to-scale technology. Each input earns a return according to its marginal product. The poverty trap does not arise from insufficient productivity but from a production function that induces a low labor share of output (relative to the capital share). Since the overlapping generation structure forces immediate consumption of all income from capital, only the young generation saves and provides tomorrow's capital stock. (Similar phenomena certainly exist in economies with a less extreme consumption schedule.) It is obvious that government policy with respect to capital control, which hurts consumers in the short-run, benefits society in the long-run by escaping the poverty trap.

It is of interest to note that our stability analysis of stochastic economies avoids a linear approximation around some associated deterministic steady state. This method is widely used in modern macroeconomics (Marimon and Scott 1999, Taylor and Uhlig 1990) although it has to be applied with caution because its validity has not yet been established rigorously.

The remainder of the paper is as follows. Section 2 introduces the model, section 3 presents the mathematical framework and the main result, and section 4 illustrates our findings by means of a numerical example.

\section{The Model}

Consider a Diamond (1965) overlapping generations economy with stochastic aggregate production where the technology exhibits constant returns to scale. A single homogeneous good, which can be either consumed or used as capital input, is produced from capital and labor in any period. Production is subject to random factors, modeled by an ergodic process.

Let $k_{t}$ denote the capital intensity at the beginning of period $t$. Then $f\left(k_{t}, z_{t}\right)$ is the output intensity which depends on the realization of the ergodic stochastic process $z_{t}$. We assume a standard neoclassical production function $f(k, z)$, i.e. continuously differentiable and strictly concave in $k \geq 0$, and $f(0, z)=0$ for every possible realization of the exogenous variable $z_{t}$.

Markets are perfectly competitive, capital and labor earn their marginal products in all states of nature, i.e. the interest rate is given by $r_{t}=f^{\prime}\left(k_{t}, z_{t}\right)$ 
and the wage rate is $w_{t}=f\left(k_{t}, z_{t}\right)-k_{t} f^{\prime}\left(k_{t}, z_{t}\right) .\left(f^{\prime}(k, z)\right.$ denotes the derivative of $f$ with respect to the first argument, $k$.) The young individual inelastically supplies one unit of labor, earning labor income $w_{t}$. This income is divided between today's consumption $c_{t}^{1}=w_{t}-s_{t}$ and savings $s_{t}$ to finance tomorrow's consumption when the individual is old (i.e. out of the labor force). Savings $s_{t}$ yield consumption $c_{t+1}^{2}=\left(1-\delta+r_{t+1}\right) s_{t}$ in the next period. The young consumer is uncertain about future interest rate $r_{t+1} \geq 0 . \quad \delta$ is the rate of depreciation. For ease of presentation we let population growth be zero and normalize the total population to one.

The lifetime preferences of an individual born in period $t$ are assumed to be represented by separable expected logarithmic utility function as

$$
\log c_{t}^{1}+\int_{\mathbb{R}_{+}} \log c_{t+1}^{2} \mu\left(d r_{t+1}\right)
$$

This specification of preferences avoids the consideration of the existence problem of a rational expectations equilibrium associated to the young agent's expectations on the future interest rate (captured by the probability measure $\mu$ ). Maximizing (1) over all feasible consumption-investment decisions, one finds the agent's optimal savings are given by

$$
s\left(w_{t}\right)=w_{t} / 2
$$

That is, the saving decision is independent of the agent's expectation. Thus we have obtained a (trivial) rational expectations equilibrium.

In a closed economy the endowment of capital per capita at the beginning of period $t+1$ is equal to the resources not consumed in the preceding period, i.e. $k_{t+1}=f\left(k_{t}, z_{t}\right)+(1-\delta) k_{t}-c_{t}^{1}-c_{t}^{2}$. The OLG structure implies that $k_{t+1}$ is given by the savings of periods $t$ young agent. The evolution of the capital intensity is governed by the stochastic law of motion

$$
k_{t+1}=\left[f\left(k_{t}, z_{t}\right)-k_{t} f^{\prime}\left(k_{t}, z_{t}\right)\right] / 2
$$

The ergodic process is assumed to take on two values $z_{t} \in\{\underline{z}, \bar{z}\}, 0<$ $\underline{z} \leq \bar{z}$. The relative frequency of each state is $p_{\underline{z}} \in(0,1), p_{\bar{z}}=1-p_{\underline{z}}$. Beside simplifying the presentation, this assumption facilitates to achieve an intuition for both, approach and results.

We restrict the further analysis to the production function

$$
f(k, z)=z \log (1+k)
$$

The following result proves that the corresponding law of motion is S-shaped for every realization of the shock. A precise definition of the S-shaped property is given in the proof of Proposition 2.1, see Appendix. 
Proposition 2.1 For each possible realization of the technology shock $z_{t}$, the law of motion

$$
k_{t+1}=h\left(k_{t}, z_{t}\right)=\frac{z_{t}}{2}\left[\log \left(1+k_{t}\right)-\frac{k_{t}}{1+k_{t}}\right]
$$

has the following properties.

(i) $h(k, z)$ is an S-shaped function of $k$ with $h(0, z)=0$.

(ii) $h(k, z)$ asymptotically grows slower than $k$ at $k=0$ and $k=\infty$. More precisely, $h^{\prime}(0, z)=\lim _{k \rightarrow \infty} h^{\prime}(k, z)=0$.

(iii) Suppose $\underline{z} \geq 9.25$. Then $h(k, \underline{z})>k$ for some $k>0$.
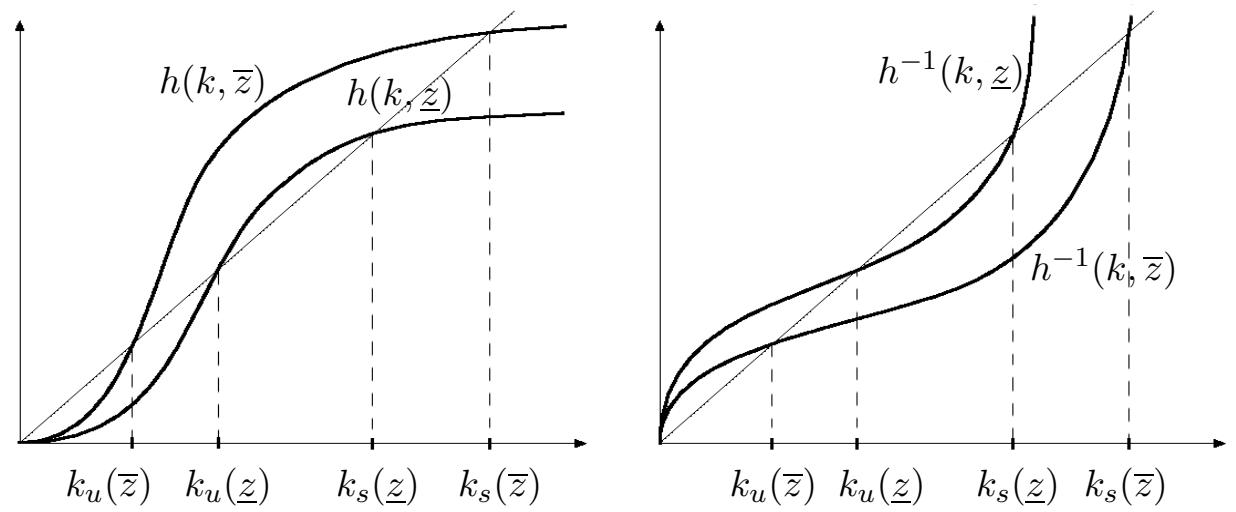

Figure 1: Sketch of an S-shaped law of motion $h(k, z)$ (left) and its inverse $h^{-1}(k, z)$ (right) for $z=\underline{z}$ and $z=\bar{z}$. The graph of the identity map is shown for reference. For constant $z, h(k, z)$ (and thus $h^{-1}(k, z)$ ) possesses three fixed points $0<k_{u}(z)<k_{s}(z)$. Zero and $k_{s}(z)$ are locally stable with domains of attraction $\left[0, k_{u}(z)[\right.$ resp. $] k_{u}(z), \infty\left[. k_{u}(z)\right.$ is locally unstable.

This result enables us to give a complete description of the evolution of the economy in the absence of production shocks, i.e. $z_{t}$ is a constant. The deterministic dynamics is characterized by two stable fixed points and one unstable fixed point, provided the condition in Proposition 2.1(iii) holds (which ensures existence of sustainable strictly positive capital stocks). Since $k \rightarrow h(k, z)$ is S-shaped and savings are not sufficient to sustain high levels of capital stocks, cf. (ii), there are exactly two strictly positive fixed points $0<k_{u}(z)<k_{s}(z)$. The first is unstable because $h(k, z)$ crosses the identity from below, i.e. for all capital stocks close to $k_{u}(z), h(k, z)<k$ for all $k<k_{u}(z)$ and $h(k, z)>k$ for all $k>k_{u}(z)$. This is immediate from the fact 
that small capital stocks cannot be sustained according to (ii). The fixed point $k_{s}(z)$ is stable because $h(k, z)$ crosses the identity from above, which also follows from (ii). Further the state of no capital $(k=0)$ is a fixed point, cf. (i). Zero is locally stable by (ii). The existence of another fixed point would violate the S-shaped property of $h(k, z)$.

The deterministic long-run behavior of any sample path is uniquely determined by the position of its initial capital stock to the unstable fixed point $k_{u}(z)$. This fixed point defines a threshold in the sense that a path of capital stocks with initial value $k_{0}$ converges toward zero if $k_{0}$ is below $k_{u}(z)$ and toward the strictly positive fixed point $k_{s}(z)$ if $k_{0}$ is above $k_{u}(z)$. The set of capital stocks $\left[0, k_{u}(z)\right.$ [ defines a poverty trap. Any event such as destruction of capital or capital drain that reduces the capital stock below the threshold $k_{u}(z)$ sends the economy in the poverty trap which, in the longrun, leads to the state of no capital. Impoverishment of the economy is the consequence. The positive content of this observation is that any supply of outside capital which leads to a capital stock larger than $k_{u}(z)$ achieves the prosperity state $k_{s}(z)$. A repayment of debt is possible without depletion of capital if the capital stock after any payment exceeds $k_{u}(z)$, i.e. avoids the poverty trap. A more detailed analysis is provided in the stochastic case.

The deterministic dynamics is the same as in Azariadis and Drazen (1990). In their paper, however, the poverty trap is due to insufficient productivity which prevents sustainability of small capital stocks. Increasing returns to scale, an externality of the aggregate capital stock, ensures that higher capital stocks (but not exceeding $k_{s}(z)$ ) can be sustained.

In the present model the technology permits any capital stock below $k_{s}(z)$ to be sustained, i.e. the constant returns-to-scale technology is sufficiently productive to avoid poverty. Extraction of capital by the capital owners for consumption and small labor-share in production for small capital stocks leads to the existence of a poverty trap. No externality is present.

\section{The Analysis}

We employ random dynamical systems theory - a brief sketch of this framework is provided below. The reader is referred to Arnold (1998) for additional information, see also Schenk-Hoppé (2001). A random dynamical system provides a mathematical description of dynamical systems that are subject to random perturbations. It consists of two ingredients: a model of the perturbation (here an ergodic dynamical system) and a model of the system perturbed by noise (here an invertible smooth mapping on $\mathbb{R}_{+}$). 
Let $(\Omega, \mathcal{F}, \mathbb{P}, \theta)$ denote the ergodic dynamical system corresponding to the exogenous shock, i.e. $\Omega=\{\underline{z}, \bar{z}\}^{\mathbb{Z}}$ the space of two-sided infinite sequences with values in $\{\underline{z}, \bar{z}\}, \mathcal{F}$ the Borel- $\sigma$-algebra, $\mathbb{P}$ the probability measure generated by the marginal distributions, and the map $\theta: \Omega \rightarrow \Omega$, $\theta(\omega(\cdot)) \equiv \omega(1+\cdot)$ the left-shift on $\Omega$. $\mathbb{P}$ is ergodic with respect to $\theta$. Denote by $\mathcal{F}^{t}$ the sub- $\sigma$-algebra of $\mathcal{F}$ generated by all histories $\left(\ldots, \omega_{t}\right)$. The stochastic component of the economy is given by a map $z: \Omega \rightarrow\{\underline{z}, \bar{z}\}$ that is $\mathcal{F}^{0}$-measurable. The state of the environment in period $t$ is given by $z\left(\theta^{t} \omega\right)$ which is measurable with respect to the history up to time $t$. The assumption on the relative frequency of each state means that the empirical measure satisfies $\lim _{T \rightarrow \infty} 1 / T \sum_{t=0}^{T} \mathbf{1}_{\underline{z}}\left(z\left(\theta^{t} \omega\right)\right)=p_{\underline{z}}$ (analogously for $\bar{z}$ ).

Denote by $\varphi(t, \omega, k)$ the state of the economy at time $t$ that is governed by the stochastic law (3), i.e.

$$
\varphi(t, \omega, k)=h\left(\cdot, z\left(\theta^{t-1} \omega\right)\right) \circ \ldots \circ h(k, z(\omega))
$$

and $\varphi(0, \omega, k)=k$.

The family of maps $\varphi(t, \omega, k)$ is called the random dynamical system generated by (5). That is, $\varphi: \mathbb{N} \times \Omega \times \mathbb{R}_{+} \rightarrow \mathbb{R}_{+},(t, \omega, k) \mapsto \varphi(t, \omega, k) \equiv$ $\varphi(t, \omega) k$ is a measurable map such that $\varphi(0, \omega)=\operatorname{id}_{\mathbb{R}_{+}}$and $\varphi(s+t, \omega)=$ $\varphi\left(t, \theta^{s} \omega\right) \circ \varphi(s, \omega)$ for all $s, t \in \mathbb{N}$ and for all $\omega \in \Omega$. These properties replace the flow property of a deterministic dynamical system generated by a map.

The main tool in the study of the dynamics of the stochastic economy is the concept of a random fixed point. The existence is proved here using a random version of the Banach fixed point theorem Schmalfuss $(1996,1998)$ that has been applied to the stochastic Solow model in Schenk-Hoppé and Schmalfuss (2001).

Definition 3.1 $A$ random fixed point of the random dynamical system $\varphi$ generated by $h$ is a random variable $k^{\star}: \Omega \rightarrow \mathbb{R}_{+}$such that almost surely

$$
k^{\star}(\theta \omega)=\varphi\left(1, \omega, k^{\star}(\omega)\right) \equiv h\left(k^{\star}(\omega), z(\omega)\right) .
$$

A random fixed point $k^{\star}$ is called stable if (for almost every $\omega$ ) there exists an open set $N(\omega)$ with $k^{\star}(\omega) \in N(\omega)$ such that for all $k \in N(\omega)$, $\lim _{t \rightarrow \infty}\left|\varphi(t, \omega, k)-k^{\star}\left(\theta^{t} \omega\right)\right|=0$. The set of all initial values $k$ that are attracted by $k^{\star}$ is called its domain of attraction.

$A$ random fixed point $k^{\star}$ is called unstable if $\left|\varphi(t, \omega, k)-k^{\star}\left(\theta^{t} \omega\right)\right| \nrightarrow 0$ as $t \rightarrow \infty$ for all $k$ in some neighborhood of $k^{\star}(\omega)$.

A random fixed point fulfills $k^{\star}\left(\theta^{t} \omega\right)=\varphi\left(t, \omega, k^{\star}(\omega)\right)$ for all $t$ by (7), i.e. $k^{\star}\left(\theta^{t} \omega\right)$ is a sample path whose state at time $t$ only depends on the realization 
of the exogenous perturbation. Moreover, it is a stationary process because the distribution of $k^{\star}\left(\theta^{t} \omega\right)$ is independent of time. If the shock is a constant, (7) becomes the definition of a fixed point of a map.

In order to avoid the discussion of degenerate cases, we make a nondegeneracy assumption on the exogenous stochastic component that excludes periodic (cyclic) perturbations. It is assumed that

(A) For all $z, z^{\prime} \in\{\underline{z}, \bar{z}\}, 0<\mathbb{P}\left\{z\left(\theta^{t} \omega\right)=z^{\prime} \mid z\left(\theta^{t-1} \omega\right)=z\right\}<1$.

This condition is fulfilled, e.g., if the stochastic perturbation is i.i.d. or, more generally, a Markov process with strictly positive transition matrix.

Theorem 3.1 The dynamics of the economy governed by the S-shaped stochastic law of motion (5) is as follows.

(i) Zero is an asymptotically stable fixed point. All capital stocks $k<$ $k_{u}(\bar{z})$ converge to the state of no capital.

(ii) Suppose $\underline{z} \geq 9.25$ and

$$
p_{\underline{z}} \log h^{\prime}\left(k_{s}(\underline{z}), \underline{z}\right)+p_{\bar{z}} \log h^{\prime}\left(k_{s}(\underline{z}), \bar{z}\right)<0
$$

Then there exists a unique stable random fixed point $k_{s}^{\star}(\omega) \in\left[k_{s}(\underline{z}), \infty[\right.$. All capital stocks $k>k_{u}(\underline{z})$ converge to the sample path $t \mapsto k_{s}^{\star}\left(\theta^{t} \omega\right)$.

(iii) Suppose in addition that (defining $K_{u}=\left[k_{u}(\bar{z}), k_{u}(\underline{z})\right]$ )

$$
p_{\underline{z}} \min _{k \in K_{u}} \log h^{\prime}(k, \underline{z})+p_{\bar{z}} \min _{k \in K_{u}} \log h^{\prime}(k, \bar{z})>0
$$

Then there exists an unstable random fixed point $k_{u}^{\star}(\omega) \in\left[k_{u}(\bar{z}), k_{u}(\underline{z})\right]$. Moreover, the domains of attraction of the stable random fixed point zero and $k_{s}^{\star}(\omega)$ are given by $\left[0, k_{u}^{\star}(\omega)\left[\right.\right.$ and $\left[k_{u}^{\star}(\omega), \infty[\right.$, respectively.

This result on the dynamics of the stochastic economy (5) with an Sshaped law of motion for any realization of the exogenous shock can be interpreted as follows.

Zero is a poverty trap in the sense that the economy starting with a (sufficiently) small capital stock experiences a decreasing sequence of capital stocks which eventually leads to the no-capital state. This is local stability of the fixed point zero.

For a sufficiently high initial stock of capital the economy converges toward the sample path $t \rightarrow k_{s}^{\star}\left(\theta^{t} \omega\right)$ of capital stocks which is determined by the (locally stable) random fixed point $k_{s}^{\star}(\omega)$. In the short run, the convergence phenomenon will dominate the behavior of the sample path of capital. Its degree of observability will be the higher the more distinct initial stock and $k_{s}^{\star}(\omega)$. In the long run, the behavior of $k_{s}^{\star}\left(\theta^{t} \omega\right)$ dominates. The 
sample path of capital will then experience permanent fluctuations which are determined by the sequence of exogenous perturbations. Capital thus exhibits business cycle dynamics caused by exogenous technology shocks.

If assumption (9) is satisfied, a very detailed statement on the critical threshold of initial capital can be made. The first result is that only two long-run phenomena are stable (and thus observable) in this economy. Either poverty or business cycle dynamics is actually observed in the long run. Moreover, the levels of initial capital stocks leading to the one or the other long-run scenario are uniquely determined by a separating critical threshold of initial capital. If the initial capital stock is above $k_{u}^{\star}(\omega)$, the path of capital converges to the business cycle. An initial capital stock below $k_{u}^{\star}(\omega)$ eventually leads to poverty.

The threshold $k_{u}^{\star}(\omega)$ depends on the sequence of shocks. But it is not observable even with an infinite history of observations because its state at any period in time depends on the future realizations of the shock. This property follows from the existence proof. A close inspection of the proof shows that $k_{u}^{\star}(\omega)$ can be constructed as the limit $\varphi^{-1}(t, \omega, k)$ as $t \rightarrow \infty$ (with $k \in] k_{u}(\bar{z}), k_{u}(\underline{z})\left[\right.$ ). Thus $k_{u}^{\star}(\omega)$ "contains" all future information on the shocks. While the actual critical level at time $t, k_{u}^{\star}\left(\theta^{t} \omega\right)$ cannot be inferred from past data, the distribution of the threshold can be determined from the structure of the economy. The distribution (or density) function of the threshold $k_{u}^{\star}(\omega)$ provides valuable information on the probability of success of an intervention. A detailed explanation follows.

Theorem 3.1(iii) ensures that $k_{u}^{\star}(\omega) \in K_{u}=\left[k_{u}(\bar{z}), k_{u}(\underline{z})\right]$ (for all $\omega$ ). The density of the random variable $\omega \rightarrow k_{u}^{\star}(\omega)$ (which is identical in all periods in time) has thus a support in $K_{u}$. The exact shape of density depends on the statistical properties of the exogenous shock. However, three important conclusions on the effect of a supply of outside capital can be drawn from this observation. First, the effect of outside capital is uncertain. It may or may not move the economy out of the poverty trap. An intervention that raises the capital stock to $k$ is successful with probability equal to the event that $k$ is higher than the unstable random fixed point $k_{u}^{\star}(\omega)$. The probability of success is increasing in the post-intervention capital stock. Second, any transfer payment that keeps the capital stock below $k_{u}(\bar{z})$ cannot be successful. With certainty, i.e. with probability one, the economy does not escape the poverty trap. Third, any lump-sum payment that raises total capital above $k_{u}(\underline{z})$ is a successful intervention in that it with certainty eventually leads to the business cycle regime.

An immediate consequence of Theorem 3.1 is that the economic aggregates output, consumption, and real wage also converge to the sample 
path of a stable random fixed point, if the capital stock does so. For instance, in the business cycle regime the random fixed points for output and real wage are given by $y_{s}^{\star}(\omega) \equiv f\left(k_{s}^{\star}(\omega), z(\omega)\right)=z(\omega) \log \left(1+k_{s}^{\star}(\omega)\right)$ and $w_{s}^{\star}(\omega) \equiv z(\omega)\left[\log \left(1+k_{s}^{\star}(\omega)\right)-k_{s}^{\star}(\omega) /\left(1+k_{s}^{\star}(\omega)\right)\right]$, respectively.

It is of interest to relate the random dynamical system analysis of the stochastic economy to the Markov equilibrium approach. We start by detailing the implications of Theorem 3.1 on the existence of Markov equilibria.

Suppose the production shock is independent and identically distributed. Then the stable random fixed point $k_{s}^{\star}(\omega)$ gives rise to a stationary measure (Markov equilibrium) by $\rho=\mathbb{E} k_{s}^{\star}(\omega)$, i.e. $\rho(B)=\mathbb{P}\left\{k_{s}^{\star}(\omega) \in B\right\}$. The transition probability is determined by $(5)$ as $P(k, B)=\mathbb{P}\{\omega \mid h(k, \omega) \in B\}$. The probability measure $\rho$ fulfills $\rho(B)=\int_{R_{+}} P(k, B) \rho(d k)$ since $h\left(k_{s}^{\star}(\omega), \omega\right)=$ $k_{s}^{\star}(\theta \omega)$ and $\omega \rightarrow k_{s}^{\star}(\omega)$ depends only on past information, i.e. is measurable with respect to the $\sigma$-algebra $\mathcal{F}^{0}$. Zero (more precisely the Dirac measure at zero) is another Markov equilibrium.

Both Markov equilibria zero and $\rho$ are stable in the sense that the empirical measure of a sample path with initial capital stock in $k<k_{u}(\bar{z})$, resp. $k>k_{u}(\underline{z})$ is identical to the respective Markov equilibrium. (Often the property is referred to as ergodic.) This is an immediate consequence of the convergence results (i) and (ii) in Theorem 3.1 and the ergodic theorem. For instance, for all $k>k_{u}(\underline{z})$

$$
\lim _{T \rightarrow \infty} \frac{1}{T} \sum_{t=0}^{T} \mathbf{1}_{B}(\varphi(t, \omega, k))=\lim _{T \rightarrow \infty} \frac{1}{T} \sum_{t=0}^{T} \mathbf{1}_{B}\left(k_{s}^{\star}\left(\theta^{t} \omega\right)\right)=\rho(B)
$$

$\mathbf{1}_{B}(k) \in\{0,1\}$ is the indicator function with value 1 if and only if $k \in B$.

These two Markov equilibria can also be obtained by the standard stable sets approach as applied for related models in Wang $(1993,1994)$. However, it is important to point out that using that method no more precise information on the domains of attraction can be given. In particular this implies that the study of the effect of supply of outside capital is limited to those events which happen with certainty.

The unstable random fixed point $k_{u}^{\star}(\omega)$ does not define a Markov equilibrium because it is not measurable with respect to the past (but with respect to the future, as explained above). This fact also follows from assertion (iii) in Theorem 3.1. All capital stocks below, resp. above, $k_{u}^{\star}(\omega)$ converge toward zero, resp. $k_{s}^{\star}\left(\theta^{t} \omega\right)$. Therefore the support of $k_{u}^{\star}(\omega)$ has non-empty intersection with the domains of attraction of zero and $k_{s}^{\star}(\omega)$. This contradicts the assumption that the distribution of $k_{u}^{\star}(\omega)$ is a Markov equilibrium. 


\section{Numerical Example}

To illustrate the concept and the main result, a numerical simulation is provided. We let $\underline{z}=10$, and $\bar{z}=15$. The exogenous stochastic process is i.i.d. with equal probability on both states.

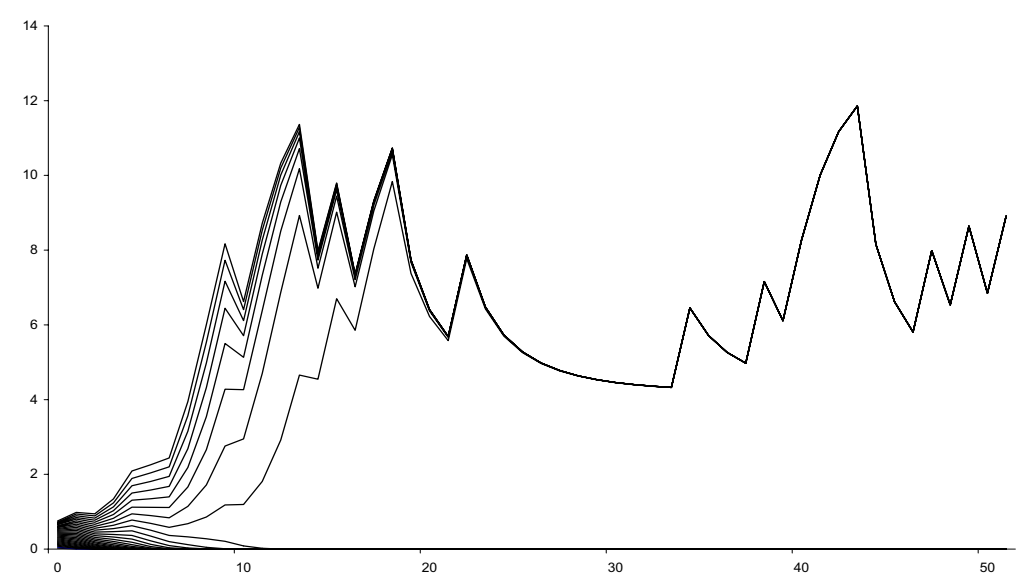

Figure 2: Sample paths $\varphi(t, \omega, k)$ for different initial capital stocks $k$ (the sequence of shocks is the same for each sample paths).

One finds $k_{u}(\underline{z}) \approx 1.1380, k_{s}(\underline{z}) \approx 4.2215, k_{u}(\bar{z}) \approx 0.4346$, and $k_{s}(\bar{z}) \approx$ 12.6550. Validity of the contraction conditions (8) and (9) can be checked numerically. Thus Theorem 3.1 applies in its full generality.

Figure 2 depicts a collection of sample paths of capital stocks. For this particular sequence of shocks the state of the unstable random fixed point at time zero is $k_{u}^{*}(\omega) \approx 0.56$. The paths corresponding to initial values above this critical threshold converge to the path $t \rightarrow k_{s}^{*}\left(\theta^{t} \omega\right)$ of the stable random fixed point $k_{s}^{*}(\omega)$ (business cycle regime). Paths with initial capital stock lower than $k_{u}^{*}(\omega)$ converge to zero (poverty trap).

Figure 3 depicts the density of both stable and unstable random fixed point. The density of the critical value $k_{u}^{\star}(\omega)$ that separates poverty trap and business cycle regime is approximated using 300.000 runs. Each run consists of an approximation of $k_{u}^{\star}(\omega)$ for one sample path of the shock using the fact that initial capital stocks below (above) $k_{u}^{\star}(\omega)$ converge to zero $\left(k_{s}^{\star}\left(\theta^{t} \omega\right) \geq k_{s}(\underline{z})\right)$. It is important to repeat that the density of $k_{u}^{\star}(\omega)$ does not correspond to a Markov equilibrium. The success probability of a lump-sum transfer can be read off from Figure 3 (left). For instance, if the capital stock is 0.645 then the chances to avoid the poverty trap is $50 \%$. 

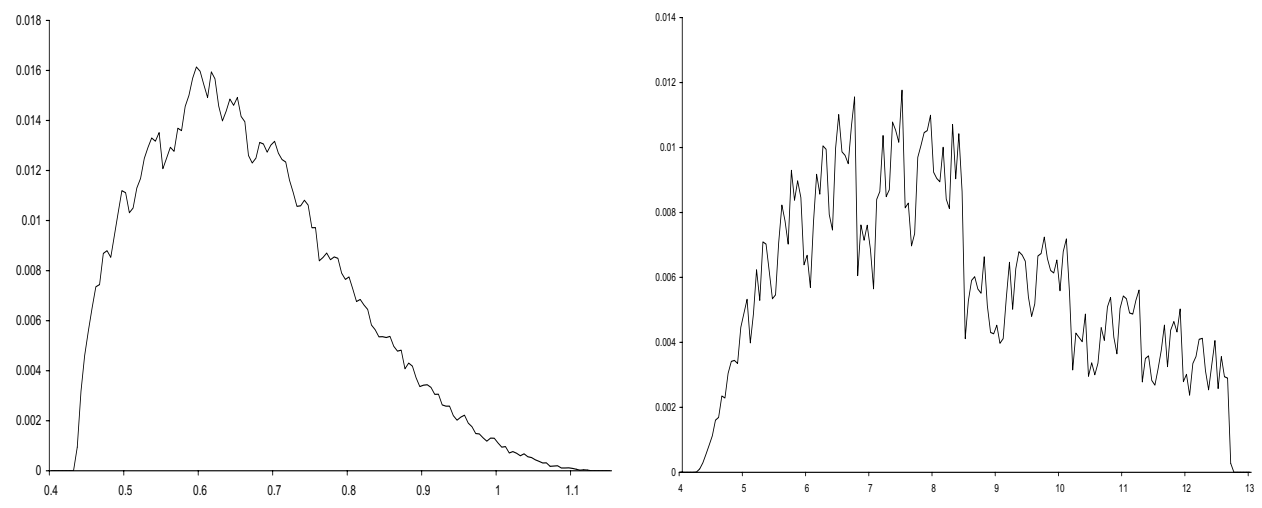

Figure 3: Densities of the threshold $k_{u}^{\star}(\omega)$ (left) and the stable random fixed point $k_{s}^{\star}(\omega)$ (right).

Increasing the capital stock to 0.845 raises this probability to $90 \%$.

The density of the stable random fixed point $k_{s}^{\star}(\omega)$ that determines fluctuations in the business cycle regime is calculated on the basis of $10^{7}$ iterations. The roughness of the density is due to the two-state shock which also causes strong changes in the capital stock in Figure 2.

\section{Appendix}

Proof of Proposition 2.1. To prove (i) recall that a function $g$ (assumed to be twice continuously differentiable) is called S-shaped if (i) $g$ is strictly increasing (i.e. $g^{\prime}(k)>0$ ), (ii) $g$ is convex up to a certain argument (inflection point) and then concave (i.e. $g^{\prime \prime}(k)>0$ for all $k<\tilde{k}$ and $g^{\prime \prime}(k)<0$ for all $k>\tilde{k})$.

The derivative of $h(k, z)$ with respect to the capital stock is given by $h^{\prime}(k, z)=(z / 2) k /(1+k)^{2}$, and the second derivative is given by $h^{\prime \prime}(k, z)=$ $(z / 2)(1-k) /(1+k)^{3}$. It is obvious from these expressions that $k \rightarrow h(k, z)$ is $\mathrm{S}$-shaped. $h(0, z)=0$ is trivially true.

Property (ii) is also immediate from the expression for $h^{\prime}(k, z)$. And (iii) follows from checking numerically that $h(2.1625, \underline{z})>2.1625$ if $\underline{z} \geq 9.25$. Since $h(k, \bar{z}) \geq h(k, \underline{z})$, the proof is complete.

Proof of Theorem 3.1. If $\bar{z}=\underline{z}$ the evolution of the economy follows the deterministic law discussed before. Thus assertions (i)-(iii) are proved. Let $\bar{z}>\underline{z}$ in the following. 
(i): Zero is a deterministic fixed point. Stability of zero follows from the fact that (1) locally convergence to zero is exponentially fast (even with arbitrarily large rate since the derivative $\left.h^{\prime}(0, z) \equiv 0\right)$, and (2) the capital stock $k_{t}$ is strictly monotonically decreasing for all $k_{0}<k_{u}(\bar{z})$ under the map $h(k, \bar{z})$ and, therefore, under $h(k, \underline{z}) \leq h(k, \bar{z})$ as well.

The proofs of (ii) and (iii) rely on the method laid out in Schenk-Hoppé and Schmalfuss (2001), Theorem 4.2. There a stochastic Banach fixed point theorem is used to prove existence of a stable random fixed point. While (ii) is straightforward, (iii) requires a novel idea to make use of the same procedure to prove existence of an unstable random fixed point.

Note that the assumption $\underline{z} \geq 9.25$ together with Proposition 2.1(iii) ensures that $h(k, \underline{z})$ and $h(k, \bar{z})$ each have two strictly positive fixed points (one unstable and one stable) with $0<k_{u}(\bar{z})<k_{u}(\underline{z})<k_{s}(\underline{z})<k_{s}(\bar{z})$.

In order to prove (ii) we have to show that (1) $K_{s}:=\left[k_{s}(\underline{z}), \infty[\right.$ is forward-invariant, (2) $h$ is uniformly contracting on $K_{s}$, and (3) the sample path of every initial capital stock $k_{0}>k_{u}(\underline{z})$ enters $K_{s}$ in finitely many steps.

Forward invariance (i.e. $h\left(K_{s}, z\right) \subset K_{s}$ for $z=\underline{z}, \bar{z}$ ) follows from $h(k, z) \geq$ $k_{s}(\underline{z})$ for all $k \geq k_{s}(\underline{z})$ and $z=\underline{z}, \bar{z}$. Uniform contraction means that $\mathbb{E} \sup _{k \in K_{s}} \log h^{\prime}(k, z)<0$. Since $h(k, \underline{z})$ is concave for $k \in K_{s}$ (otherwise $k_{s}(\underline{z})$ would not be a stable fixed point of the S-shaped map $\left.h(k, \underline{z})\right)$, linearity in $z$ ensures that $h(k, \underline{z})$ also concave on $K_{s}$. Thus, for $k \in K_{s}$, both $h^{\prime}(k, \underline{z})$ and $h^{\prime}(k, \bar{z})$ take on its maximum at $k=k_{s}(\underline{z})$. The contraction therefore is equivalent to $\mathbb{E} \log h^{\prime}\left(k_{s}(\underline{z}), z\right)<0$, but this is assumption (8). These two properties, forward invariance and uniform contraction, ensure existence of a stable random fixed point in $K_{s}$.

To prove that the domain of attraction contains all capital stocks $k>$ $k_{u}(\underline{z})$, it suffices to observe that sample paths on $\left[k_{u}(\underline{z}), k_{s}(\underline{z})\right]$ are strictly monotonically increasing. Since $\underline{z}<\bar{z}$, there is a (deterministic) set $\tilde{K}:=$ $\left[\tilde{k}, k_{s}(\underline{z})\right]$ with $\tilde{k}<k_{s}(\underline{z})$ such that $h(k, \bar{z})>k_{s}(\underline{z})$ for all $k \in \tilde{K}$. Pick any initial capital stock $k$ in this set, then after a finite number of steps (and for any sample path of the shock) $k_{t} \in \tilde{K}$. Non-degeneracy of the shock and the monotonicity property on $\left[k_{u}(\underline{z}), k_{s}(\underline{z})\right]$ ensure that the sample path enters $K_{s}$ in a finite number of steps.

The key to prove (iii) is to analyze the random dynamical system $\psi$ generated by the inverse maps $h^{-1}(k, z)$. The inverse of $h$ is well-defined thanks to strict monotonicity. The random dynamical systems $\psi$ and $\varphi$ are related by $\psi(t, \omega, k)=\varphi^{-1}(t, \omega, k)$. The proof proceeds in three steps. First, it is shown that $K_{u}:=\left[k_{u}(\bar{z}), k_{u}(\underline{z})\right]$ is forward-invariant for $h^{-1}(k, z)$. Second, uniform contraction of $h^{-1}(k, z)$ on this set is ensured. This yields 
existence of a random fixed point $k_{u}^{\star}(\omega) \in K_{u}$. The fixed point is stable for $h^{-1}(k, z)$ with domain of attraction $\left(0, k_{s}(\underline{z})\right)$. Third, it is proved that under the original random dynamical system all initial states $k>k_{u}^{\star}(\omega)$ converge to $k_{s}^{\star}(\omega)$ and all states $k<k_{u}^{\star}(\omega)$ converge to zero.

It is simple to see that $h^{-1}(k, z)$ is increasing in $k$ and decreasing in $z$, cf. Figure 1. These properties imply, $k_{u}(\bar{z})=h^{-1}\left(k_{u}(\bar{z}), \bar{z}\right) \leq h^{-1}(k, \bar{z}) \leq$ $h^{-1}(k, \underline{z}) \leq h^{-1}\left(k_{u}(\underline{z}), \underline{z}\right)=k_{u}(\underline{z})$. Thus, $K_{u}:=\left[k_{u}(\bar{z}), k_{u}(\underline{z})\right]$ is forwardinvariant, i.e. $h^{-1}\left(K_{u}, z\right) \subset K_{u}$ for $z=\underline{z}, \bar{z}$.

To show that $h^{-1}(k, z)$ is uniformly contracting on $K_{u}$ one needs to ensure

$$
\mathbb{E} \sup _{k \in K_{u}} \log \partial_{k} h^{-1}(k, z)<0
$$

Using that $\partial_{k} h^{-1}(k, z)=1 / h^{\prime}(x, z)$ with $x=h^{-1}(k, z)$, one obtains the sequence of estimates $\sup _{k \in K_{u}} \log \partial_{k} h^{-1}(k, z)=\sup _{x \in h^{-1}\left(K_{u}, z\right)} \log \left(1 / h^{\prime}(x, z)\right)$ $\leq \sup _{k \in K_{u}} \log \left(1 / h^{\prime}(k, z)\right)=-\inf _{k \in K_{u}} \log h^{\prime}(k, z)\left(\right.$ recalling $h^{-1}\left(K_{u}, z\right) \subset$ $K_{u}$.) Note that the capital stock for which this infimum is taken on typically depends on the shock.

This estimate leads to a sufficient condition for (10). One has that

$$
\mathbb{E} \inf _{k \in K_{u}} \log h^{\prime}(k, z)>0
$$

implies uniform convergence of $h^{-1}$ on $K_{u}$. But (11) is equivalent to assumption (9). Summarizing our findings, we can state that there exists a unique stable random fixed point $k_{u}^{\star}(\omega)$ in $K_{u}$ for the random dynamical system $\psi$. Clearly, $k_{u}^{\star}(\omega)$ is a random fixed point for the random dynamical system $\varphi$. Considerations analogous to (ii) show that $k_{u}^{\star}(\omega)$ attracts (under $\psi$ ) all initial capital stocks in $\left(0, k_{s}(\underline{z})\right.$ ). In particular every capital stock $k \in\left(0, k_{u}(\bar{z})\right)$ is attracted to the sample path of $k_{u}^{\star}(\omega)$. This implies the convergence property $k_{u}^{\star}(\omega)-\psi\left(t, \theta^{-t} \omega, k\right) \rightarrow 0$ (and the difference is always non-negative). Therefore, every $k \in\left[k_{u}(\bar{z}), k_{u}^{\star}(\omega)\right)$ eventually becomes less than $k_{u}(\bar{z})$ under $\varphi(t, \omega)$ as $t$ tends to infinity. In the long run $\varphi(t, \omega, k) \in\left(0, k_{u}(\bar{z})\right)$ which is in the domain of attraction of the stable fixed point zero. Thus $\left[k_{u}(\bar{z}), k_{u}^{\star}(\omega)\right)$ is found to be in the domain of attraction of zero. Similarly one finds that $\left(k_{u}^{\star}(\omega), k_{s}(\underline{z})\right]$ is in the domain of attraction of the stable random fixed point $k_{s}^{*}(\omega)$.

\section{References}

Arnold, L., 1998. Random Dynamical Systems. Springer-Verlag. New York. 
Azariadis, C., Drazen, A., 1990. Threshold Externalities in Economic Development. Quarterly Journal of Economics 105, 501-526.

Diamond, P., 1965. National Debt in a Neoclassical Growth Model. American Economic Review 55, 1126-1150.

Marimon, R., Scott, A., (Eds.), 1999. Computational Methods for the Study of Dynamic Economies. Oxford University Press. Oxford U.K.

Schenk-Hoppé, K.R., 2001. Random Dynamical Systems in Economics. Stochastics and Dynamics 1, 63-83.

Schenk-Hoppé, K.R., Schmalfuss, B., 2001. Random Fixed Points in a Stochastic Solow Growth Model. Journal of Mathematical Economics $36,19-30$.

Schmalfuss, B., 1996. A Random Fixed Point Theorem Based on Lyapunov Exponents. Random \& Computational Dynamics 4, 257-268.

Schmalfuss, B., 1998. A Random Fixed Point Theorem and the Random Graph Transformation. Journal of Mathematical Analysis and Applications 225, 91-113.

Taylor, J.B., Uhlig, H., 1990. Solving Nonlinear Stochastic Growth Models: A Comparison of Alternative Solution Models. Journal of Business and Economic Statistics 8, 1-18.

Wang, Y., 1993. Stationary Equilibria in an Overlapping Generations Economy with Stochastic Production. Journal of Economic Theory 61, $423-435$.

Wang, Y., 1994. Stationary Markov Equilibria in an OLG Model with Correlated Production Shocks. International Economic Review 35, 731-744. 